\title{
MOLECULAR ENVELOPES OF YOUNG AND PROTO PNE
}

\section{S. DEGUCHI}

\author{
Nobeyama Radio Observatory, \\ National Astronomical Observatory, \\ Minamimaki, Minamisaku, Nagano 384-13, Japan
}

\begin{abstract}
I review recent results of mm-wave interferometric observations of molecular envelopes of young and proto planetary nebulae. Spatial resolutions of mm-wave interferometers now reach from one to a few arcseconds; together with line observations reveale very small structures in molecular envelopes of planetary nebulae. These small scale structures are probably caused by an instability of the radiation-driven, gas-dust outflow. Implications of these observations and theories are discussed.
\end{abstract}

\section{Introduction}

The recent improvements in interferometric techniques enable us to study very tiny structures in the envelopes of planetary nebulae (PNe). Spatial resolutions achieved by $\mathrm{mm}$-wave interferometers at Nobeyama, Owens Valley, Hat Creek and Grenoble now reach a few arcseconds (for previous summary, see Deguchi \& N-Q-Rieu 1991). With this high resolution, a number of very small scale structures in the molecular envelopes of $\mathrm{PNe}$ are now being revealed. Their physical relation to the morphology of $\mathrm{PNe}$ is quite interesting from the view point of the dynamical evolution of the envelope. A relation of these high-resolution observations with recent HST results must be considered. In this paper, I first describe several examples of smallscale structures which were observed by radio interferometers. About two dozens of interferometric observations of $\mathrm{PNe}$ with spatial resolutions better than 5" have been published till today. Table 2 shows a list of published interferometric observations of $\mathrm{PNe}$ and proto-planetary nebulae ( $\mathrm{PPNe})$. Observations of AGB stars as IRC+10216, CIT6 etc. are omitted from this list; for observations of these AGB stars, see papers by Bieging \& Nguyen- 


\section{S. Deguchi}

Q-Rieu (1988b; 1989), Bieging \& Tafalla (1993), Takano et al. (1992), Lucas et al. (1992), Dayal et al. (1993, 1995), and Gensheimer et al. (1995).

TABLE 1. Interferometric observations of PNe and PPNe

\begin{tabular}{|c|c|c|}
\hline Source name & Molecules (line) & Reference \\
\hline CRL 2688 & $\mathrm{CO}(\mathrm{J}=1-0)$ & Masson et al. 1985 \\
\hline CRL 2688 & ${ }^{13} \mathrm{CO}(\mathrm{J}=1-0)$ & Yamamura et al. 1995, 1996 \\
\hline CRL 2688 & $\mathrm{HCN}(\mathrm{J}=1-0)$ & Bieging \& N-Q-Rieu 1988a \\
\hline CRL 2688 & $\mathrm{HC}_{3} \mathrm{~N}, \mathrm{HNC}, \mathrm{SiS}$ & N-Q-Rieu \& Bieging 1990 \\
\hline CRL 2688 & $\mathrm{NH}_{3}, \mathrm{HC}_{5} \mathrm{~N}$ & Nguyen-Q-Rieu et al. 1986 \\
\hline CRL 2688 & $\mathrm{HC}_{5} \mathrm{~N}, \mathrm{SiS}$ & Lucas 1994 \\
\hline CRL 618 & $\mathrm{CO}(\mathrm{J}=1-0)$ & Shibata et al. 1993 \\
\hline CRL 618 & $\mathrm{CO}(\mathrm{J}=1-0)$ & Hajian et al. 1996 \\
\hline CRL 618 & ${ }^{13} \mathrm{CO}(\mathrm{J}=1-0)$ & Yamamura et al. 1994 \\
\hline CRL 618 & $\mathrm{CS}(\mathrm{J}=2-1)$ & Hajian et al. 1995 \\
\hline CRL 618 & $\mathrm{NH}_{3}(3,3)$ & Martin-Pintado et al. 1995 \\
\hline CRL 618 & $\mathrm{NH}_{3}(3,3)$ & Bachiller et al. 1993 \\
\hline NGC7027 & $\mathrm{CO}(\mathrm{J}=1-0)$ & Bieging et al. 1991 \\
\hline NGC7027 & $\mathrm{HCO}^{+}(\mathrm{J}=1-0)$ & Deguchi et al. 1992 \\
\hline NGC7027 & $\mathrm{HCO}^{+}(\mathrm{J}=1-0)$ & Likkel et al. 1992 \\
\hline IRAS $21282+5050$ & $\mathrm{CO}(\mathrm{J}=1-0)$ & Shibata et al. 1989 \\
\hline IRAS $21282+5050$ & $\mathrm{CO}(\mathrm{J}=1-0)$ & Meixner et al. 1996 \\
\hline M1-7 & $\mathrm{CO}(\mathrm{J}=1-0)$ & Shibata et al. 1994 \\
\hline M1-92 & $\mathrm{CO}(\mathrm{J}=1-0)$ & Bujarrabal et al. 1994 \\
\hline M1-92 & ${ }^{13} \mathrm{CO}(\mathrm{J}=1-0)$ & Bujarrabal et al. 1996 \\
\hline
\end{tabular}

\section{Interferometric Observations of Proto-Planetary Nebulae}

The well-known, best studied example is CRL 2688, the Egg Nebula, which exhibits a bipolar structure in optical and near-infrared images (Ney et al. 1975). A number of interferometric observations have been made for this nebula. Yamamura et al. $(1995,1996)$ mapped this nebula in the ${ }^{13} \mathrm{CO} \mathrm{J}=1$ 0 line with the Nobeyama Millimeter Array (4" resolution) and the 45-m telescope (16" resolution). The combined map of interferometer and singledish data shows that the density distribution of the envelope is quite spherically symmetric, through there is some high density elongation to the south.

The combination of the single-dish and interferometeric data is quite important; interferometers are sensitive to small high-brightness features but often lose the flux from weak, extended components; often the lost flux amounts to $2 / 3$ of the total flux of the line emission. Single-dish data (with 
less spatial resolution) cover these short-spacing fringes which cannot be obtained from interforometers. They are quite complimentary to correctly map the total flux of emission.

From the modeling of the envelope using the Large Velocity Gradient (LVG) approximation, we can obtain the gas density in the envelope and hence derive the variation of mass loss rate of the last 1000 years from the central star. Accoding to Yamamura et al. (1996), the mass loss rate varied over the last few hundred years from $1 \times 10^{-4}$ to $3 \times 10^{-4} \mathrm{M}_{\odot} \mathrm{yr}^{-1}$ and seems to have stopped stop approximately 600 years ago.

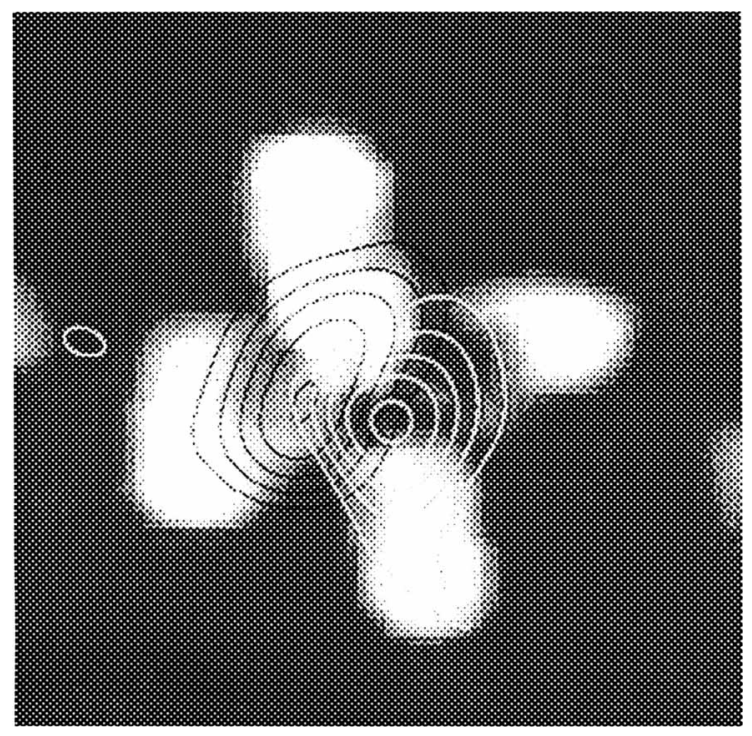

Figure 1. A overlay of ${ }^{13} \mathrm{CO}$ high velocity emission (contours) on the $\mathrm{H} 2$ emission (picture: $20 " \times 20$ ") at the infrared in CRL2688. The white contour on the right is the redshifted component and the black contour on the left is the blueshifted. (the ${ }^{13} \mathrm{CO}$ map is from Yamamura et al. 1995; the H2 picture from Dr. C. Skinner).

The other interesting feature is the high-velocity flow, which is resolved in this ${ }^{13} \mathrm{CO}$ observation. The direction of the ${ }^{13} \mathrm{CO}$ high-velocity flow seems to be oriented in a direction slightly different from the direction of the bipolar axis. Figure 1 shows an overlay of the map of the ${ }^{13} \mathrm{CO}$ high velocity flows on the infrared image of the $\mathrm{H}_{2}$ vibrational emission. The $\mathrm{H}_{2}$ emission comes from the 4 points, two of which coincide with the direction of the bipolar axis the other two hot spots of $\mathrm{H}_{2}$, however, seem to look like shocks at the inner edges of the disk. The "quadri-polar structure" of 


\section{S. Deguchi}

hydrogen-molecule emission is clearly seen in this figure. The positions of the ${ }^{13} \mathrm{CO}$ high velocity flow are slightly different from the positions of the near-infrared molecular hydrogen emission. The direction of the ${ }^{13} \mathrm{CO}$ flow does not coincide exactly with any of these $\mathrm{H}_{2}$ features.

Recently, Kasuga et al.(1996) have analysed data of the CS J=1-0 and 2-1 transitions in CRL 2688 taken at Nobeyama. Figure 2 shows the integrated-intensity map of CS. The overall feature is somewhat similar to ${ }^{13} \mathrm{CO}$; the outer envelope looks spherically symmetric. However, at the inner core, emission in the $\mathrm{J}=2-1$ transition exhibits an elongation close to the optical bipolar axis but slightly misaligned. The abundance of CS seems to be slightly increased at this part.

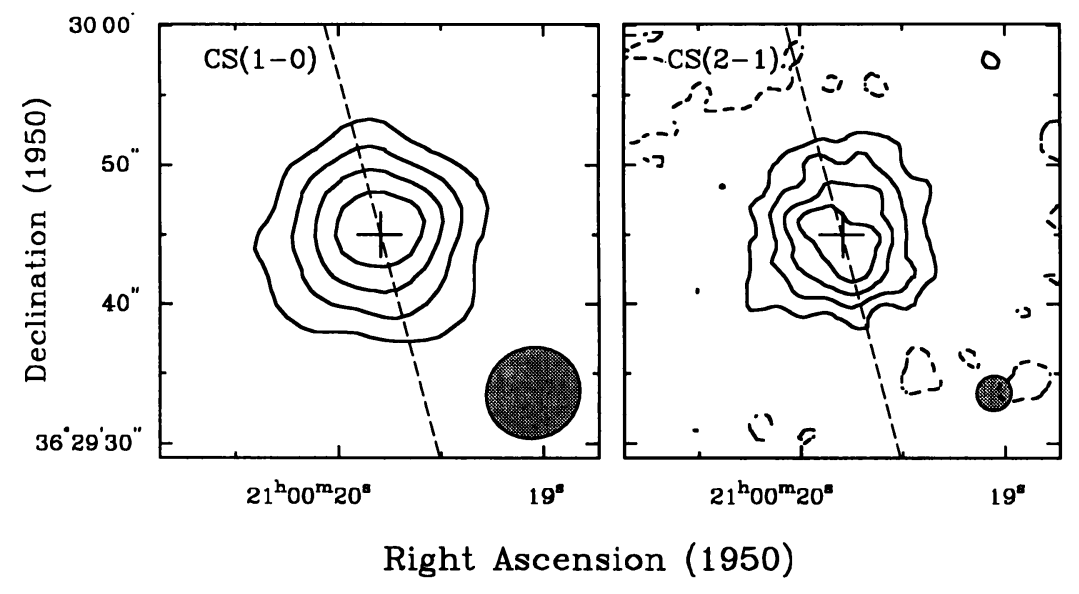

Figure 2. The integrated intensity map of CRL2688 in the CS J=1-0 line (left pannel) and in the CS $\mathrm{J}=2-1$ line (right pannel). The broken line shows the direction of the bipolar axis.

Another representative protoplanetary is CRL 618. Shibata et al. (1993) and Yamamura et al. (1994) mapped this nebula in the ${ }^{12} \mathrm{CO}$ and ${ }^{13} \mathrm{CO}$ $\mathrm{J}=1-0$ lines with Nobeyama Interferometer and obtained velocity channel maps in each lines. The optical bipolar axis is oriented approximately in East-West in this nebula. However, we can see a number of irregular structures which seem not to be oriented to the bipolar structure. From the ${ }^{12} \mathrm{CO}$ and ${ }^{13} \mathrm{CO}$ data, we can obtain the density and temperature distributions in the entire 3-dimensional space of the envelope if the velocity field is assumed. It is well known that ${ }^{12} \mathrm{CO}$ is a measure of gas kinetic temperature 


\section{MOLECULAR ENVELOPES OF PNE}

and ${ }^{13} \mathrm{CO}$ is more or less a measure of gas density. Therefore if these two data are combined, we can obtain the three-dimensional density and temperature structure of the envelope of PPNe (Yamamura et al. 1994). The results show that the gas kinetic temperature increases along the bipolar axis. The other irregular structures are probably due to irregularity of the kinetic temperature and not due to the irregularity of gas density.

Recently, Hajian et al. (1995) made a high-resolution mapping of the core of CRL 618 in the CS $\mathrm{J}=2-1$ line and found that CS emission is elongated in the direction approximately by 45 degree to the optical bipolar axis. They also found an absorption feature in CS line. These irregularities and the orientation of CS emission agree with the results obtained by Shibata et al. (1994) in CO lines.

Martin-Pintado et al. (1995) have analysed $\mathrm{NH}_{3}$ data in CRL 2688. They also mapped the continuum emission at $23 \mathrm{GHz}$ from this nebula. The size of the continuum emission in this nebula is about 0.5 " and the continuum is elongated along the optical bipolar axis. They found a difference of $\mathrm{NH}_{3}$ absorption lines in the different parts of the continuum. Therefore the ammonia cloud has clumps of about 0.1 ", or less than about $10^{15} \mathrm{~cm}$. This observation suggests that there are tiny clumpy irregular structures in envelopes of PPNe.

NGC 7027 is a very bright young planetary nebula. CO emission is extended to about one arc minute (Bieging et al. 1991), indicating a large ionized molecular envelope. Deguchi et al. (1990) found that intensity of the $\mathrm{HCO}^{+} \mathrm{J}=1-0$ line is quite strong, and it is stronger than the intensity of the $\mathrm{HCN} \mathrm{J=1-0} \mathrm{line.} \mathrm{The} \mathrm{mapping} \mathrm{observations} \mathrm{of} \mathrm{HCO}^{+}$emission (Deguchi et al. 1992; Likkel 1992) revealed that the $\mathrm{HCO}^{+}$molecules are concentrated at the periphery of the ionized gas. Model calculations (Yamamura \& Deguchi 1994) indicate that $\mathrm{HCO}^{+}$is located in a torus with the axis inclined by about $60^{\circ}$ from the line of sight. The $\mathrm{HCO}^{+}$molecule is considered to work as a catalyzer in ion-molecule reations and the abundance of this molecule influences very strongly the chemistry of the outer molecular envelope (see Deguchi 1995).

Radio observations discovered a number of small-scale structures in envelopes of PPNe. The most important structure among these is, of course, the high velocity flow. The orientation of the high-velocity flow does not coincide with the optical bipolar axis and it appears to have a "precession" motion. The "precession", is not exactly a precession of a rotating body, but the features observed by various molecular lines, if connected, look like a curved string coming out from a rotating body. It reminds us of a neutron star with the rotating dipole magetic field misoriented from the rotation axis. 


\section{S. Deguchi}

\section{Stability of the gas-dust outflow}

Recently, Sahai et al. (1995) found with the HST that the dust envelope of CRL 2688 consists of many arclets or thin dust shells. The thickness of the shell is approximately $10^{16} \mathrm{~cm}$. Narrow double beams of the scattering light were also seen. The shell can be observed only in the tangential direction at thickest part of the spherically symmetric shell and other part of the shell cannot be seen probably because of the lower optical depth of the shell.

What is the origin of these thin shells? Is the gas-dust flow intrinsically unstable or does the mass loss terminate periodically? A similar question is whether or not this phenomenon is common in all PPNe and masslosing AGB stars. Another question is whether or not the gas has a similar distribution as the dust. If this is so how do the interpretations of radio and infrared data change? Especially, the estimation of the mass loss rate of this source has to be altered because previous estimations assumed a smooth distribution of the gas density.

There are two possible explanations on this phenomenon. One is the recuring He-shell flash, however, then the time between two helium-flashes is too short: judging from the separation of the shell in CRL 2688, the flash must occur once every 200 years. If we adopt the He-shell-flash period-coremass relation given by Paczynski (1975), the core mass must be about 1.15 $\mathrm{M}_{\odot}$. In this case, the initial mass of the star must be more than $10 \mathrm{M}_{\odot}$ and the luminosity at the AGB phase must more than $3 \times 10^{4} \mathrm{~L}_{\odot}$. It is somewhat improbable for AGB stars to have such a large mass.

A more plausible explanation is an instability of a radiation-driven gasdust flow. An excellent review on this problem was given before by Morris (1992). The standard linear perturbation analysis is the common method to discuss the stability. Here, I present a simple version of the linear perturbation analysis.

First we give the steady state solution and then put some perturbation on it and see if the perturbation is growing or damping. In the previous analysis by Morris (1992), the thermal pressure term is negleted. However, this term leads to a special instability. All parameters are assumed to be a function of radius.

For the analysis, we use equation of motion for the gas,

$$
\partial v_{g} / \partial t+v_{g} \partial v_{g} / \partial r=G M\left(F_{r}-1\right) / r^{2}-\rho_{g}^{-1} \partial P / \partial r
$$

and equations of continuity for the gas and for the dust (see Kwok 1975):

$$
\partial \rho_{g} / \partial t+r^{-2} \partial\left(r^{2} \rho_{g} v_{g}\right) / \partial t=0,
$$

and

$$
\partial \rho_{d} / \partial t+r^{-2} \partial\left[r^{2} \rho_{d}\left(v_{g}+v_{d f t}\right)\right] / \partial t=0
$$




\section{MOLECULAR ENVELOPES OF PNE}

where $\rho_{g}$ and $\rho_{d}$ are the mass densities of gas and grains, respectively, and $v_{g}$, the expanding velocity of the gas, and $v_{d f t}$, the drift velocity of grains to the gas. Here $F_{r}$ is the radiation pressure per unit mass in unit of the gravitational force $G M / r^{2}$. The equation of motion for the dust grains is integrated. In practice, it is assumed that the radiation pressure on grains is in equilibrium with the gas friction on grains:

$$
\pi a^{2} Q L /\left(4 \pi c r^{2}\right)=\alpha \pi a^{2} v_{d f t}\left(v_{t}^{2}+v_{d f t}^{2}\right)^{1 / 2},
$$

where $a, Q, \alpha$, and $L$ are the grain radius, the efficiency of radiation scattering, the sticking probablity, and the luminosity of the star. This is verified because the time scale of motion grain is about 100 times shorter than the gas dynamical time scale. We make the perturbed quantities proportional to $\exp [i(k r-\omega t)]$ and obtain a dispersion relation, a third order equation in $\omega$. The dispersion relation is quite simple when we neglect the higher order terms of the inverse of the wave number, $k$, because we are interested in the large scale perturbation. Only the second order terms of $k^{-1}$ are retained. In this case, it depends only on 4 non-dimensional parameters. Most important parameters among them are $p$, which is a measure of wave number of perturbation $(=h / k)$, and the parameter $g$, which is a square of sound velocity $\left(=v_{s}\right)$ devided by dust drift velocity $\left(=v_{d f t}\right)$. The dispersion relation is,

$$
Z^{3}+(p q i-1) Z^{2}-g Z+g-p i+B p^{2}=0
$$

where

$$
Z=\left(\omega-k v_{g 0}+i \partial v_{g 0} / \partial r\right) / k v_{s 0}
$$

Here the quantities with subscript 0 indicate the unperturbed state. When the imaginary part of $\omega$ is positive, the perturbation is growing and the system is unstable. Equation (5) can be solved numerically. An inspection of the plot of the imaginary part of $Z$ as a function of two parameters, $p$ and $g$, reveals that unstable point occurs when the parameter $g$ is close to unity.

The system becomes unstable when the sound velocity is nearly equal to the dust drift velocity. It can be understood by the following way (see Figure 3). Suppose that a density irregularity occurs in the outflowing gas. The grain speed is influenced by the gas density. When the gas is thin, the dust drift velocity becomes higher because the gas friction is less, and when the gas density becomes higher at the other point, the grain drift velocity become smaller because there is more friction by gas. Therefore, the gradient in the dust drift velocity is produced. This gradient causes the density-wave of grains. If we subtract the average drift velocity of grains, the motion of grains causes concentration of grains at one point(see Figure 


\section{S. Deguchi}

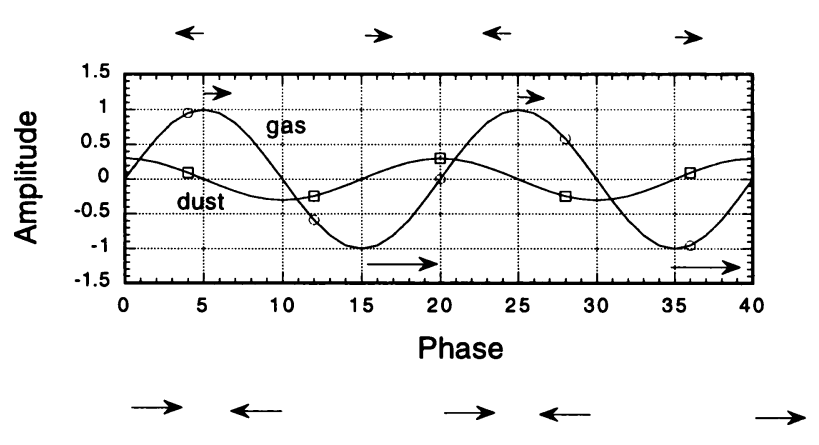

Figure 3. Density waves of the gas and grains. The flow becomes unstable when the drift velocity of grains to the gas is equal to the sound velocity. The arrows in the frame indicate the grain speed. The length is proportional to the magnitude. The arrows above the frame indicate the relative speed to the average dust motion (the drift-velocity gradient). The arrows under the frame indicate the radiation pressure acting on the gas through grains (the average is subtracted). That caused the compression of the gas.

3 ) and dissipation of grains at another point. Therefore, the dust density wave become like the curve drawn in Figure 3.

When we consider the dust density wave, the dense part of dust receives more radiation pressure and the rarefied part less. Therefore, the gas is pushed more where the grain density is higher. This compresses the gas more at the dense part of the gas.

For this instability to occur, the perturbation speed of the gas must be equal to the dust drift velocity. Mathematically, the parameter, $g$, is then equal to unity. The other important conclusion of this two-stream instability is that the phase of the perturbation of the gas is by $90^{\circ}$ advanced to the phase of dust perturbation. This analysis predict that the gas density wave can be seen very close to the dust shell but by a quarter of wavelength in advance. This effect might be detectable in the Hubble Space Telescope.

We have calculated the unperturbed state and obtained the point where the instability starts. In the model of CRL 2688, the mass loss rate is taken to be $2 \times 10^{-4} \mathrm{M}_{\odot} \mathrm{yr}^{-1}$. When the drift velcity of the gas exceeds the sound velocity, the radiation pressure driven flow becomes unstable at the 


\section{MOLECULAR ENVELOPES OF PNE}

radius of about $5 \times 10^{15} \mathrm{~cm}$. We have calculated $\omega$ in unit of crossing time scale, i.e., the radius divided by the gas velocity at each radius. If this is above unity, the perturbation can develope in enough short time. In this case, near the unstable point, it grows quite rapidly; about 1000 times of the crossing time scale. The unstable wavelength is typically, one tenth of the radius. In the high mass-loss case, the unstable point starts relatively at the outer envelope. In the case of the mass loss rate of $3 \times 10^{-5} \mathrm{M}_{\odot} \mathrm{yr}^{-1}$, the unstable point start at the radius of $3 \times 10^{14} \mathrm{~cm}$. In this case, the wavelength of perturbation is very small and difficult to observe. In the case of low mass-loss rates, this instability makes very small scale structures.

From these still preliminary studies, we can draw some conclusions on the instabilities of radiation-driven flow. The observable large-scale instablity can develop only when with the mass-loss rate is very high. This is due to the requirement of unstable condition that the grain drift velocity is comparable with the sound velocity. This fact well explains the case of CRL 2688. Mastrodemos et al. (1996) recently investigated this process further including the thermal balance of the gas in the wind.

\section{Conclusion}

Molecular line observations with radio interferometers reach now the resolution of a few to one arcsecond. With these observations, the central cores of protoplanetaries are going to be resolved. It turns out that the highvelocity flow is not exactly oriented in the direction of the optical bipolar axis.

Further complex structures are seen and we are still do not understand the origin of these fine structures. The radiation-driven flow is unstable and it is possible that the fine structures are created by this instability. With this model, we can explain the different features appearing in the different molecular lines. From these observation, it may be possible to state somewhat extreme proposition that main morphological structure of planetary nebulae were created at the protoplanetary phase.

\section{References}

Bachiller, R., Martin-Pintado, J., \& Fuente, A., 1993, ApJ, 417, L45

Bieging, J. H., \& Nguyen-Q-Rieu, 1988a, $A p J$, 324, 516

Bieging, J. H., \& Nguyen-Q-Rieu, 1988b, ApJ 329, L107

Bieging, J. H., \& Nguyen-Q-Rieu, 1989, ApJ 343, L25

Bieging, J. H. \& Tafalla, M., 1993, $A J$ 105, 576

Bieging, J. H. \& Wilner, D., \& Thronson, H., Jr., 1991, ApJ, 379, 271

Bujarabal, V., Alcolea, J., Neri, R., Grewing, M., 1994, ApJ, 436, L169

Bujarabal, V., Alcolea, J., Neri, R., \& Grewing, M., 1996, it A\&A, (preprint)

Dayal, A., \& Bieging, J. H., 1995, ApJ, 439, 996

Dayal, A., \& Bieging, J. H., 1993, ApJ, 407, L37 


\section{S. Deguchi}

Deguchi, S., 1995, in Asymmetrical Planetary Nebulae, P 132, ed. by A. Harpas and N. Soker

Deguchi, S., Izumiura, H., Kaifu, N., Mao, X., Nguyen-Q-Rieu, \& Ukita, N., 1990, ApJ, 351, 522

Deguchi, S., Izumiura, H., Nguyen-Q-Rieu, Shibata, K., Ukita, N., \& Yamamura, I., 1992, $A p J, 392,597$

Deguchi, S. \& Nguyen-Q-Rieu, 1991, Ann. Phys. Fr., 16, 413

Gensheimer, P. D., Likkel, L., \& Snyder, L. E., 1995, ApJ, 439, 445

Hajian, A. R., Phillips, J. A., \& Terzian, Y., 1995, ApJ, 446, 244

Hajian, A. R., Phillips, J. A., \& Terzian, Y., 1996, ApJ, 467, 341

Kasuga, T., Yamamura, I., \& Deguchi, S., 1996, $A \& A p$ (in press)

Kwok, S., 1975, ApJ, 198, 583

Likkel, L., 1992, ApJ, 397, L115

Lucas, R., 1994, in Astronomy with Millimeter and Submillimeter Wave Interferometry, ASP Conf. Series vol. 59, 135

Lucas, R. et al., 1992, $A \& A, \mathbf{2 6 2}, 491$

Masson et al. 1985, $A p J$, 292, 464

Martin-Pintado, J, Gaume, R., Johnston, K., \& Bachiller, R., 1995, ApJ, 446, 687

Mastrodemos, N., Morris, M, \& Caster, J., 1996, ApJ, 468, 851

Meixner, M., Welch, W. J., Likkel, L., Tafalla, M., \& Campbell, M. T., 1996, in this proceeding

Morris, M., 1992, in Mass Loss on the AGB and Beyound, P60 ed. by H. Schwartz

Ney, E. P., Merrill, K. M., Becklin, E. E., Neugebauer, G., \& Wynn-Williams, C. G., 1975, ApJ,198, L129

Nguyen-Q-Rieu, \& Bieging, J. H., 1990, ApJ, 359, 131

Nguyen-Q-Rieu, Winnberg, A., \& Bujarrabal, V., 1986, $A \& A, 165,204$

Paczynski, B., 1975, ApJ, 202, 558

Sahai, R., Trauger, J., Evans, R. W., the WFPC2 IDT, 1995, BAAS, 27, 1344

Shibata, K. M., Deguchi, S., Kasuga, T., Tamura, S., Hirano, N., \& Kemeya, O., 1994, in Astronomy with Millimeter and Submillimeter Wave Interferometry, ASP Conf. Series vol. 59, 143

Shibata, K. M., Deguchi, S., Hirano, N., \& Tamura, S., 1993, ApJ, 415, 708

Shibata, K. M., Tamura, S., Deguchi, S., Hirano, N., Kameya, O., \& Kasuga, T., 1989, $A p J, 345, \mathrm{~L} 55$

Takano, S., Saito, S., Tsuji, T., 1992, PASJ, 44, 469

Yamamura, I., \& Deguchi, S., 1994, in Astronomy with Millimeter and Submillimeter Wave Interferometry, ASP Conference Series, Vol.59, 148

Yamamura, I. Shibata, K. M., Kasuga, T., \& Deguchi, S., 1994, ApJ, 427, 406

Yamamura, I., Onaka, T., Kamijo, F., Deguchi, S., \& Ukita, N., 1995, ApJ, 439, L13

Yamamura, I., Onaka, T., Kamijo, F., Deguchi, S., \& Ukita, N., 1996, ApJ, 465, 926 